\title{
A Modified Technique for Culturing Primary Fetal Rat Cortical Neurons
}

\author{
Sui-Yi Xu, Yong-Min Wu, Zhong Ji, Xiao-Ya Gao, and Su-Yue Pan \\ Department of Neurology, Nanfang Hospital, Southern Medical University, 1838 GuangZhou Road, GuangDong, \\ GuangZhou 510515, China \\ Correspondence should be addressed to Su-Yue Pan, pansuyue82@yahoo.com.cn
}

Received 3 August 2012; Revised 30 September 2012; Accepted 30 September 2012

Academic Editor: Michael D. Coleman

Copyright ( $(2012$ Sui-Yi Xu et al. This is an open access article distributed under the Creative Commons Attribution License, which permits unrestricted use, distribution, and reproduction in any medium, provided the original work is properly cited.

The study explored a modified primary culture system for fetal rat cortical neurons. Day E18 embryos from pregnant Sprague Dawley rats were microdissected under a stereoscope. To minimize enzymatic damage to the cultured neurons, we applied a sequential digestion protocol using papain and Dnase I. The resulting sifted cell suspension was seeded at a density of 50,000 cells per $\mathrm{cm}^{2}$ onto $0.1 \mathrm{mg} / \mathrm{mL}$ L-PLL-covered vessels. After a four-hour incubation in high-glucose Dulbecco's Modified Eagle's Medium (HG-DMEM) to allow the neurons to adhere, the media was changed to neurobasal medium that was refreshed by changing half of the volume after three days followed by a complete medium change every week. The cells displayed progressively robust neurite extension, and nonneuronal-like cells could barely be detected by five days in vitro (DIV); cell growth was still substantial at 14 DIV. Neurons were identified by $\beta$-tubulin III immunofluorescence, and neuronal purity within the cultures was assessed at over $95 \%$ by both flow cytometry and by dark-field counting of $\beta$-tubulin III-positive cells. These results suggest that the protocol was successful and that the high purity of neurons in this system could be used as the basis for generating various cell models of neurological disease.

\section{Introduction}

The primary culture of fetal rat cortical neurons is widely used in cell models of many neurological disorders. However, owing to the complicated procedures involved in dissection and culture, a universally accepted protocol for their derivation has not yet been determined. Diverse techniques make it difficult to readily compare results obtained from different cell models and to repeat experiments in other laboratories. Therefore, it is essential to develop a simple and reproducible protocol for the study cell models. Here, we introduce a modified protocol for the primary culture of fetal rat cortical neurons.

\section{Materials and Methods}

2.1. Animals and Anatomy. The research procedures involving animals were approved by the Ethics Committee of Southern Medical University. SPF-class E18 Sprague Dawley pregnant rats were sacrificed by cervical dislocation. All the surgical equipment used were sterilized in an autoclave prior to use. The sacrificed pregnant rat was immersed in $75 \%$ $\mathrm{EtOH}$ for short-term disinfection after the abdominal skin was prepared. The rat was covered by a sterile towel, and the abdominal cavity was exposed with hemostatic forceps. The uterus was removed by rotating clockwise from the left lower quadrant, and the mesometrium and blood vessels were removed along the line as shown (Figure 1(a)), with care taken to maintain an intact uterus while minimizing bacterial contamination from any damaged intestines. The uterus was removed quickly and placed into a $100 \mathrm{~mm}$ sterile petri dish containing cold Hanks' balanced salt solution (HBSS) on ice (Figure 1(b)). Two elbow tweezers were used to open the uterus and transfer the fetuses into another $100 \mathrm{~mm}$ sterile petri dish containing cold HBSS on ice (Figure 1(c)). The placenta was removed, and the color, fetal movements, and number of stillbirths were recorded (Figure 1(d)). 


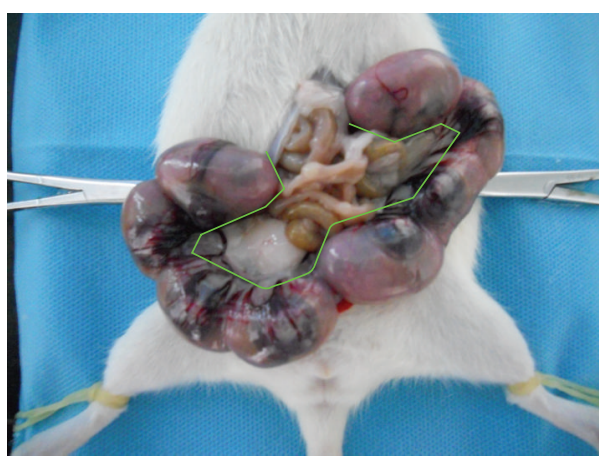

(a)

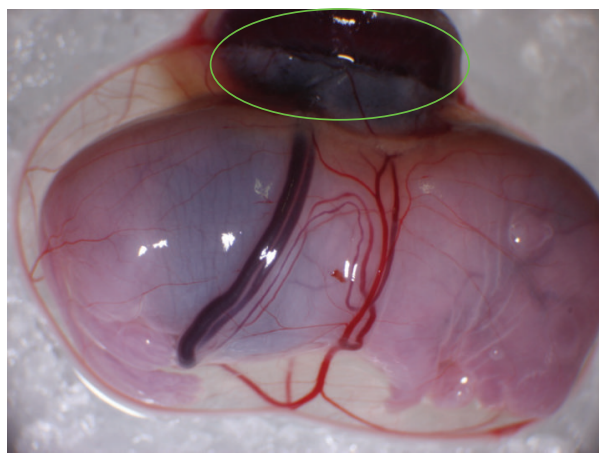

(c)

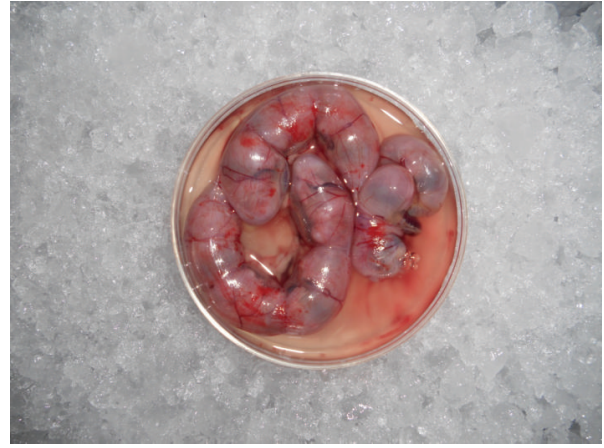

(b)

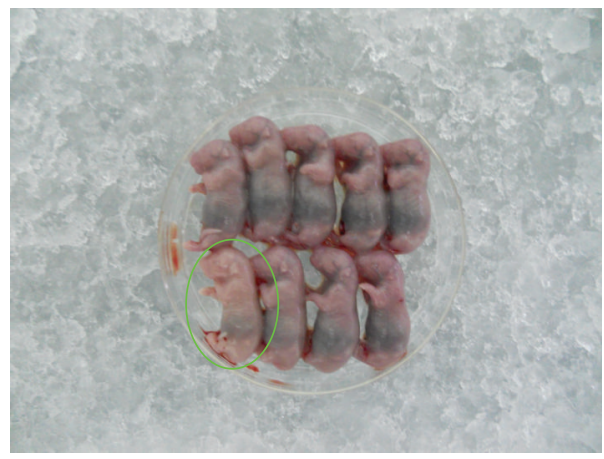

(d)

FIgURE 1: (a) The mesometrium and blood vessels were cut along the line as shown, (b) removal of the uterus, (c) removal of the placenta, (d) stillbirths recorded.

2.2. Microsurgical Anatomy under Stereoscope. All microsurgical equipment used was sterilized in an autoclave. The straight tweezers were held in the left hand to grip the neck of the fetus, while the right hand held the microsurgical elbow tweezers that were inserted into nose (forceps tip up). In a previously reported technique [1], one side of the microsurgical elbow tweezers was inserted to dissociate the tissue (Figure 2(a)), however, we found that this can cause inconsistent traction that can damage the cerebral hemispheres or the neck. Therefore, we modified the technique to insert the two legs of the tweezers simultaneously (Figure 2(b)). The expansion force of the tweezers was used to dissect with bilateral force while simultaneously removing tissue. The tweezers were then inserted at the intersection of the lambda suture to dissect the skull and dura mater (Figures 2(c) and 2(d)).

The residual skull (Figure 3(a)) and cerebral dura mater (Figure 3(b)) were removed. Some previous reports [1] used microsurgical tweezers (forceps tip up) to lift the whole brain from the front edge and then separate the cerebral cortex, hippocampus, and cerebellum (Figure 3(c)). However, the tissue of the fetal rat brain is relatively soft, and we found that lifting the brain could be difficult and may damage the tissue. Instead, we used the two legs of the microsurgical tweezers to remove the cortex rather than the whole brain (Figure 3(d)).

2.3. Dissection of the Pia Mater and Blood Vessels. Some protocols say to dissect the cerebral cortex in cold PBS or
HBSS [2, 3]. The dissection of the pia mater and blood vessels can take considerable time, and the metabolism of fetal rat neurons is very robust. Long exposure to a sugarfree environment could damage the neurons, even when kept on ice. Therefore, we rapidly transferred the dissected cerebral cortex to a $35 \mathrm{~mm}$ sterile petri dish containing cold HG-DMEM (containing 10\% fetal bovine serum (FBS)) to provide energy for metabolism (Figure 4(a)). Using the tip of a pair of microsurgical straight tweezers to hold the cortex in place, another pair of elbow tweezers was used to remove the remaining pia mater and blood vessels.

Notes. (1) Do not remove the pia mater and blood vessels from individual pieces of cortex as you dissect them. Rather, collect all of the fetal cortices quickly and remove the pia and blood vessels one-by-one in cold HG-DMEM (containing $10 \%$ FBS). (2) Take care when removing the pia mater and blood vessels (Figure 4(b)), otherwise, following cell digestion, any presence of pia or blood vessels will increase the pipetting force, which could damage the neurons. Proliferation of contaminating cells from the pia mater and blood vessels could affect the experimental results and even cause neuronal death.

2.4. Primary Neuronal Culture. Sequential digestion: the cortex is put into a sterile $35 \mathrm{~mm}$ petri dish (containing cold 


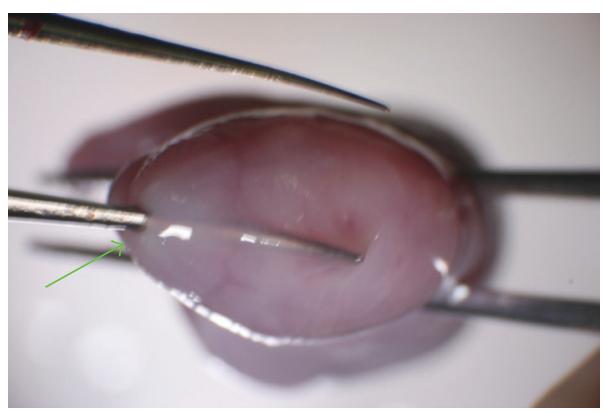

(a)

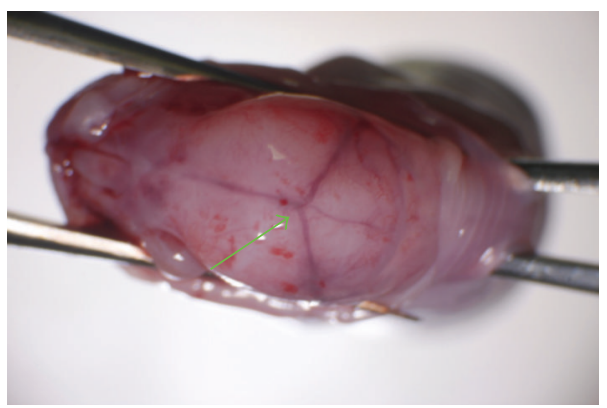

(c)

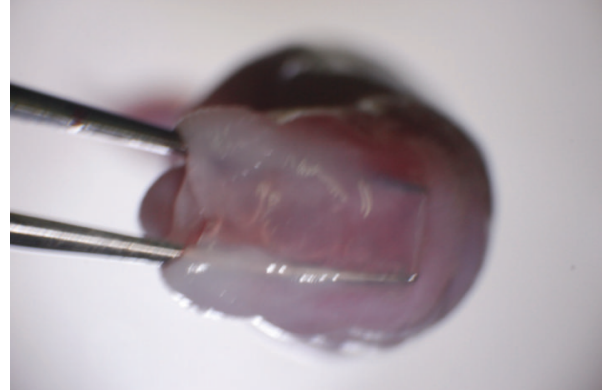

(b)

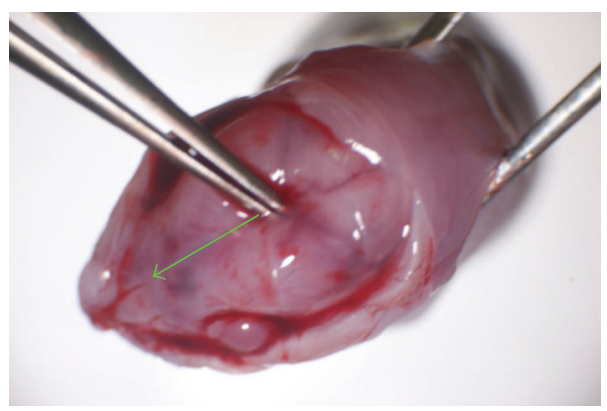

(d)

FIGURE 2: (a) Traditional approach, (b) modified approach, (c) craniotomy point and (d) dissection of skull and cerebral dura mater.

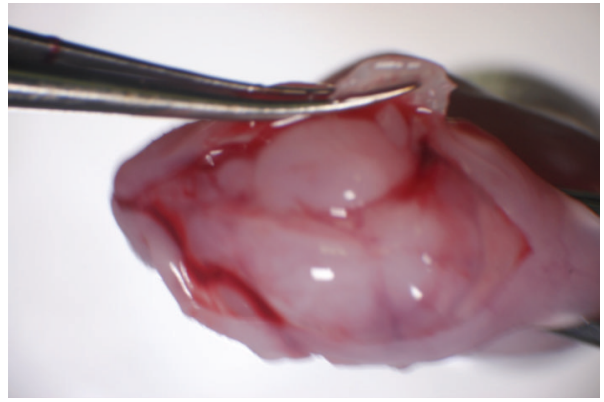

(a)

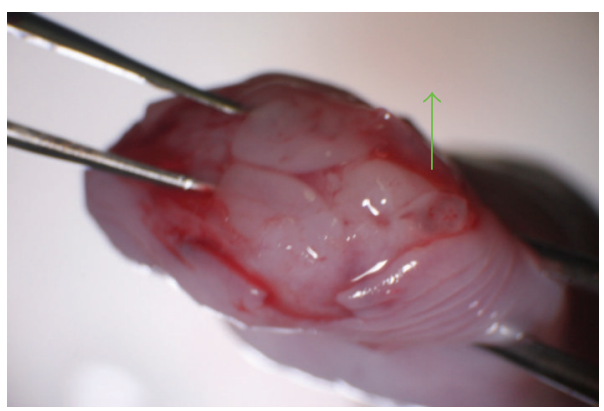

(c)

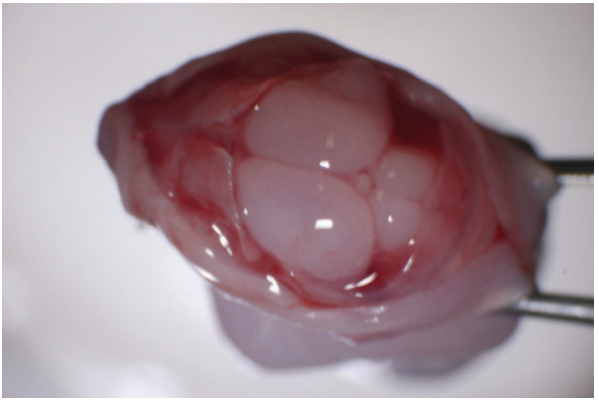

(b)

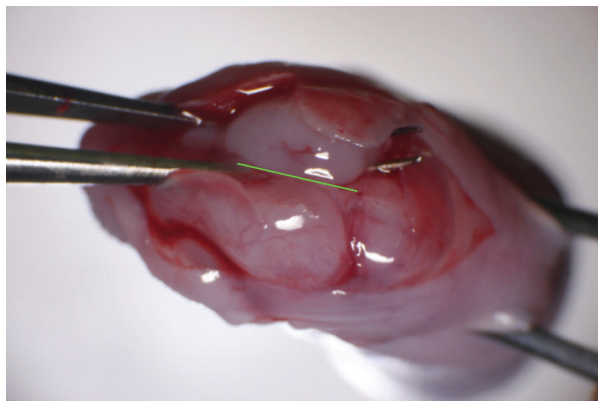

(d)

Figure 3: (a) Removal of the skull, (b) removal of the cerebral dura mater, (c) traditional approach, and (d) removal of the cortex.

FBS-free HG-DMEM) and cut into small pieces approximately $1 \mathrm{~mm}$ in length (or triturated by a fire-polished sterile Pasteur pipette). One side of the petri dish was slightly elevated to precipitate the tissue to the other side and aid in removing the culture medium. Papain $(1 \mathrm{~mL})$ dissolved in HG-DMEM $(2 \mathrm{mg} / \mathrm{mL})$ was added to the petri dish, and the tissue was digested for 30 minutes in a $37^{\circ} \mathrm{C}$ incubator. DNase I $(200 \mu \mathrm{L})$ dissolved in HBSS $(2.5 \mathrm{mg} / \mathrm{mL})$ was then added for 30 seconds. Finally, $1 \mathrm{~mL}$ of FBS was added to terminate the digestion. 


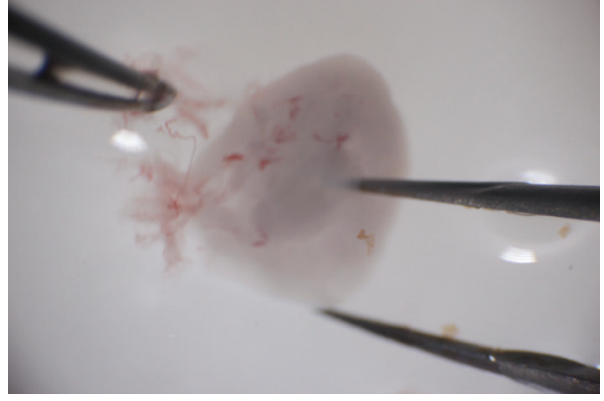

(a)

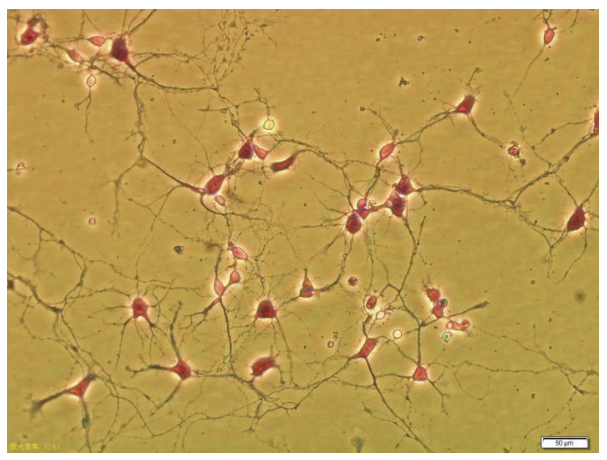

(c)

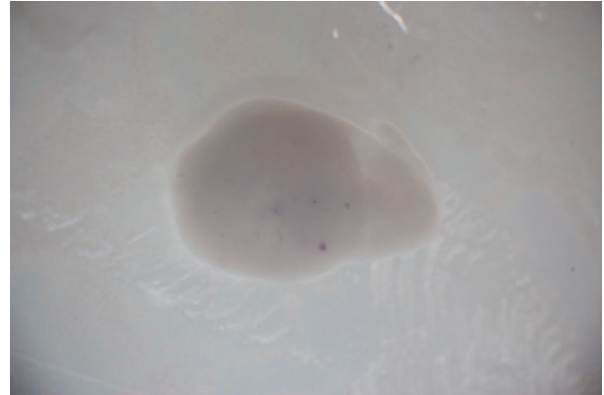

(b)

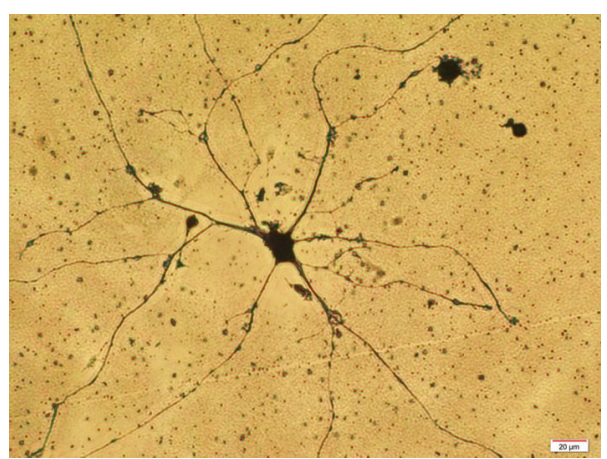

(d)

FIGURE 4: (a) Dissection of pia mater and blood vessels, (b) dissection degree, (c) 5 DIV (HE staining, bar = 50 um), and (d) 14 DIV (bar = 20 um).

Notes. Some protocols digest the tissue in centrifuge tubes $[4,5]$. The result is that digestion is insufficient because the tissue precipitates at the bottom of the tube, while the upper tissue is overdigested. Our modification is to use a sterilized, disposable $35 \mathrm{~mm}$ petri dish for digestion, allowing the papain solution to form a broad, shallow layer and keep the tissue well distributed, which leads to a uniform digestion. Some protocols have recommended $0.125 \%$ or $0.25 \%$ trypsinization; although the digestive efficiency is good, it is difficult to control making it easy to digest the tissue excessively $[6,7]$. Therefore, we have modified the procedure to a sequential digestion of papain and DNase I. Papain is mild and easy to control (we used FBS-free HG-DMEM to dissolve the papain to maintain neuronal metabolism). DNase I can break down the DNA of lysed cells that can tangle with tissue and prevent further digestion [8].

2.4.1. Preparation of Cell Suspension. The contents of the petri dish were transferred to a $15 \mathrm{~mL}$ sterile centrifuge tube. HG-DMEM was added to a volume of $10 \mathrm{~mL}$, and a sterile fire-polished Pasteur pipette was used to triturate the cells gently for about 20 times while avoiding air bubbles that could result in oxidative damage to the cells [9]. A $100 \mu \mathrm{m}$ sterile cell strainer was placed onto a $50 \mathrm{~mL}$ centrifuge tube to filter out any remaining tissue fragments after pipetting. The filtrate was centrifuged at $800 \mathrm{rpm}$ for 5 minutes, and the supernatant was removed. HG-DMEM (containing $100 \mathrm{u} / \mathrm{mL}$ penicillin/streptomycin (P/S) and 10\% FBS) was added to resuspend the cells prior to cell counting. Ideally, the cell mass should be less than $10 \%$ and the percentage of dead cells should be around $1 \%$ as revealed by $0.2 \%$ trypan blue staining.

2.4.2. Cell Seeding and Medium Replacement. L-polylysine (L-PLL) was dissolved in HBSS to a final concentration of $0.1 \mathrm{mg} / \mathrm{mL}$. Filter-sterilized ( $0.2 \mathrm{um}) \mathrm{L}-\mathrm{PLL}$ solution was added to 6 well plates or $25 \mathrm{~cm}^{2}$ culture flasks to coat their surface overnight in a $37^{\circ} \mathrm{C}$ incubator. The plates or culture flasks were washed twice with HBSS and then air-dried in a biosafety cabinet. The density of cell suspension was adjusted to a seeding density of 50,000 cells per $\mathrm{cm}^{2}$. HGDMEM was changed to neurobasal medium (containing $10 \%$ B27 supplement and $100 \mathrm{u} / \mathrm{mL}$ P/S) after four hours of incubation. The neurobasal medium was refreshed by exchanging half of the volume with fresh media three days later and then completely refreshed every week afterwards [10]. Cellular morphology was observed under an inverted phase contrast microscope. The majority of cells with round somas and a few neurites adhered well to the vessels by $4 \mathrm{~h}$ when the medium was initially changed. The cell somata show strong refractivity and stereoscopic perception; the cells exhibit progressively robust neurite extension that forms a network with neighboring neurons. Nonneural-like cells could barely be detected at 5 DIV (Figure 4(c)), cell growth remained energetic at 14 DIV (Figure $4(\mathrm{~d})$ ).

Notes. Concerning the choice of cultural vessels, 6 well plates and $25 \mathrm{~cm}^{2}$ culture flasks are optimal choices (plates with 


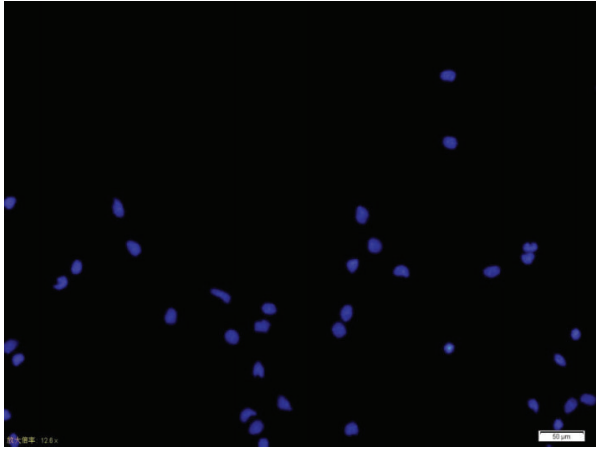

(a)

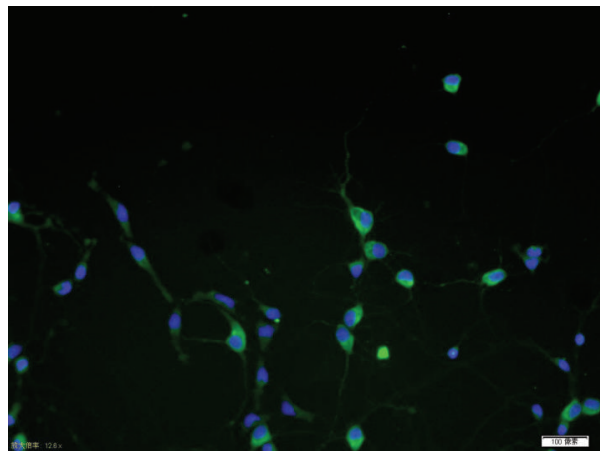

(c)

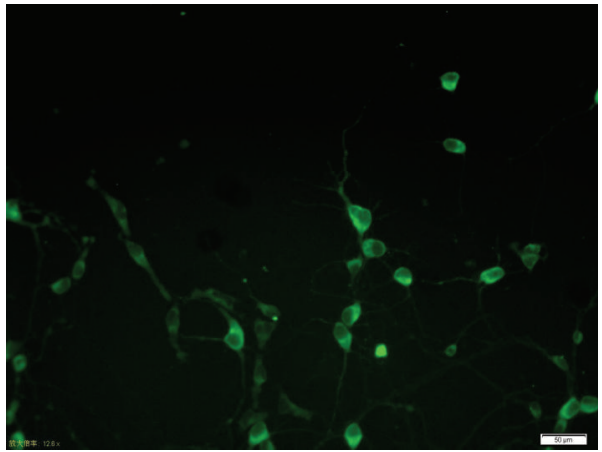

(b)

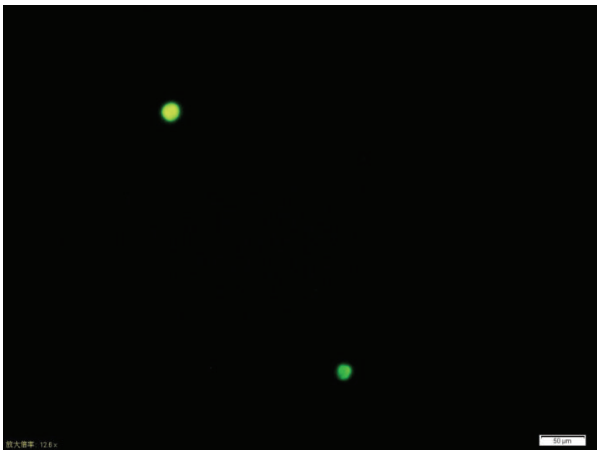

(d)

Figure 5: (a) DAPI+, (b) $\beta$-tubulin III+, (c) merge, and (d) negative control.

a U- or V-shaped bottom should not be used) because it is almost impossible to ensure a consistent cell density from the edge to the center of a plate with more than 6 wells. Differences in cell density can lead to discrepancies in culture maturity. Concerning the choice of L-PLL, its standard molecular weights for cell culture use are 70,000$15,0000,150,000-300,000$, and $>300,000$. As the molecular weight increases, the adhesive strength increases as well but so does the difficulty in getting the L-PLL to dissolve. We used a molecular weight of L-PLL of 150,000-300,000. Previously reported working concentrations differ significantly from $0.01 \mathrm{mg} / \mathrm{mL}$ to $0.25 \mathrm{mg} / \mathrm{mL}[2,3,11]$. For cell adhesion, the concentration should be kept as low as possible because LPLL is cytotoxic (PLL that is used for immunohistochemistry should not be used, because some formulations contain preservatives that will kill the cells). The seeding density also greatly influences neuronal growth. A high density leads to contact inhibition, while low density affects maturity [12]. The seeding density has been calculated as the cell number per $\mathrm{ml}$ in some reports [7]. It is not conducive to compare these results because neurons show adherent growth. Therefore, it is more appropriate to calculate the seeding area based on the area of the culture vessels [13]. Shaking the vessels in a linear motion helps to produce a uniform distribution of the cell suspension; a circular motion will cause the cells to become concentrated in the center of the vessel, resulting in nonuniform growth. We observed optimal results when the first medium replacement occurred at $4 \mathrm{~h}$. At a time earlier than $4 \mathrm{~h}$, some neurons that had not yet adhered are washed away or flow to the other side of the vessel to adhere, resulting in low or nonuniform seeding. In contract, after a period of longer than $12 \mathrm{~h}$, cell debris will also adhere firmly, which has a direct influence on neural survival and metabolism. Additionally, glial cells begin to divide after $12 \mathrm{~h}$, and at this point cytarabine must be added to inhibit glial cells and improve neural purity $[5,10]$; cytarabine is cytotoxic to neural function. Frequent medium replacement is not suitable, as changing the neurobasal medium in short intervals will remove released factors from the environment [14].

\section{Results and Discussion}

Neuronal Identification by Immunofluorescence at 5 DIV [15]. The neurons were fixed with $4 \%$ paraformaldehyde at $37^{\circ} \mathrm{C}$ for $1 \mathrm{~h}$ and then washed three times with HBSS. Primary antibody ( $1: 100$ rabbit anti-rat $\beta$-tubulin III containing BSA and triton X-100) was added to the vessel surface for a $2 \mathrm{~h}$ incubation at $37^{\circ} \mathrm{C}$, and the cells were then washed three times with HBSS. An Alexa Fluor 488-labeled goat anti-rabbit IgG $(1: 200)$ and the DNA-binding dye 4,6diamidino-2-phenylindole (DAPI) were added for a $1 \mathrm{~h}$ incubation at $37^{\circ} \mathrm{C}$ in the dark. Fluorescence images were acquired with an Olympus inverted microscope equipped with a CoolLED fluorescent light source. Nearly all of the cells were DAPI- (Figure 5(a)) and $\beta$-tubulin III-positive (Figure $5(\mathrm{~b})$ ). The determination of neuronal purity using 


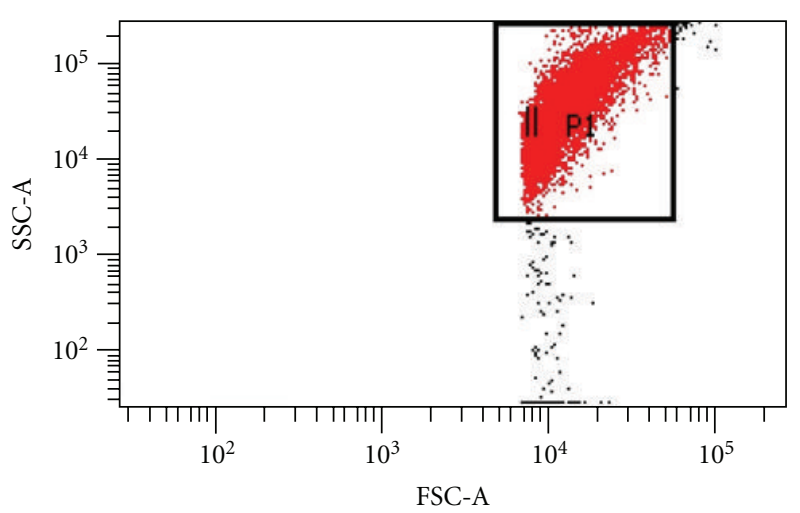

(a)

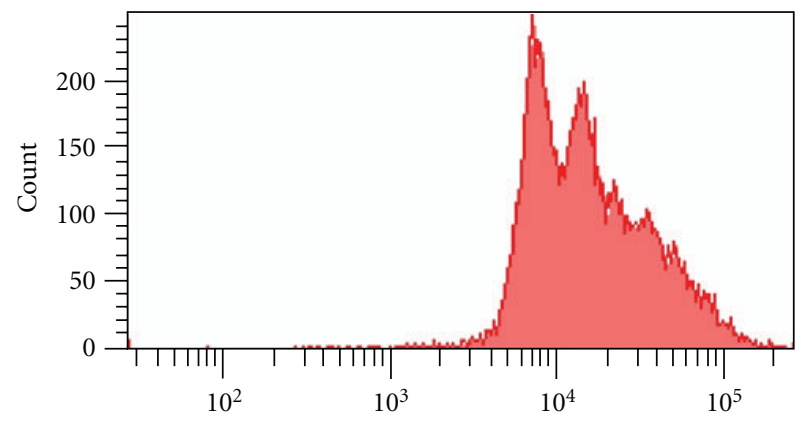

(c)

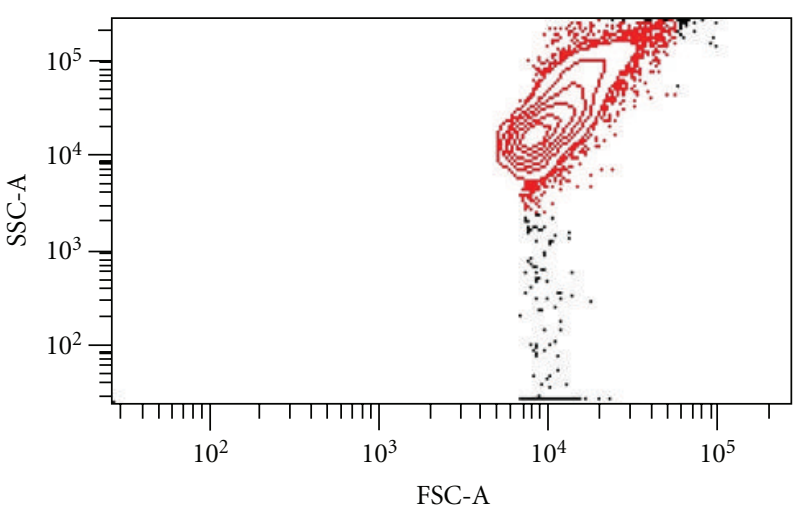

(b)

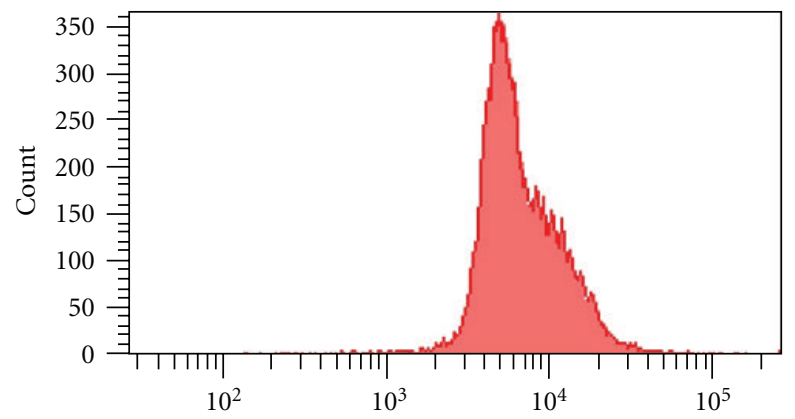

(d)

Figure 6: (a) ROI, (b) contour map, (c) $\beta$-tubulin III-A, and (d) $\beta$-tubulin III-H.

dark field imaging suggests that the percentage of $\beta$-tubulin III-immunostained neurons was over 95\% (Figure 5(c)); accordingly $96.8 \%$ were identified as neurons when assessed by flow cytometry (Figure 6).

Interestingly, we found neurons exhibit auto-fluorescence in the negative control group, regardless of whether the primary, secondary, or no antibody was added. We speculate that the nonspecific autofluorescence may come from tissue fragments or mixed cells (Figure 5(d)).

\section{Conclusion}

With the development of cell technology, primary neuronal culture has been widely applied in studying cell function, neurodevelopment, and neurological diseases. Neurons can be cocultured with endotheliocytes and astrocytes to mimic neurovascular units in vitro [16] for the purposes of studying the blood-brain barrier [17]. Every neurodevelopmental stage can be studied depending on the culture time [18]. Local neuronal plasticity can also be observed separately [19, 20]. However, the steps involved in creating primary cultures of fetal rat cortical neurons are tedious, and any mistakes made in the steps of their generation can lead to failure of that culture. Researchers all over the world constantly improve their corresponding protocols. Therefore, determining a consistent protocol is essential for the ability to compare experimental results from different laboratories. This study modifies several steps from microsurgical dissection to primary culture to generate a high-purity culture of neurons that can be the basis for various cell models used to study neurological diseases.

\section{References}

[1] T. Fath, Y. D. Ke, P. Gunning, J. Götz, and L. M. Ittner, "Primary support cultures of hippocampal and substantia nigra neurons," Nature Protocols, vol. 4, no. 1, pp. 78-85, 2009.

[2] C.-Y. Yuan, G.-S. W. Hsu, and Y.-J. Lee, "Aluminum alters NMDA receptor $1 \mathrm{~A}$ and $2 \mathrm{~A} / \mathrm{B}$ expression on neonatal hippocampal neurons in rats," Journal of Biomedical Science, vol. 18 , p. 81, 2011.

[3] C. Contreras-Jurado and A. Pascual, "Thyroid hormone regulation of APP, (beta-amyloid precursor protein) gene expression in brain and brain cultured cells," Neurochemistry International, vol. 60, no. 5, pp. 484-487, 2012.

[4] Q. Jiang, Y. W. Jiang, J. M. Wang, J. Qin, and X. R. Wu, “An improved method for primary culture of rat cortical neuron and cell identification," Journal of Peking University, vol. 41, no. 2, pp. 212-216, 2009.

[5] H. Koito and J. Li, "Preparation of rat brain aggregate cultures for neuron and glia development studies," Journal of Visualized Experiments, e1304, no. 31, 2009.

[6] M. Geissler and A. Faissner, "A new indirect co-culture set up of mouse hippocampal neurons and cortical astrocytes on microelectrode arrays," Journal of Neuroscience Methods, vol. 204, no. 2, pp. 262-272, 2012.

[7] I. Ullah, N. Ullah, M. Imran Naseer et al., "Neuroprotection with metformin and thymoquinone against ethanol-induced 
apoptotic neurodegeneration in prenatal rat cortical neurons," BMC Neuroscience, vol. 13, article 11, 2012.

[8] W.-S. Chen, C. Y. Yueh, Y. A. Huang, and E. Hwang, "An inverted method for culturing dissociated mouse hippocampal neurons," Neuroscience Research, vol. 70, no. 1, pp. 118$123,2011$.

[9] J. Harris, H. Lee, C. T. Tu, D. Cribbs, C. Cotman, and N. L. Jeon, "Preparing e18 cortical rat neurons for compartmentalization in a microfluidic device," Journal of Visualized Experiments, 305, no. 8, 2007.

[10] Y. Nakatsu, Y. Kotake, K. Komasaka et al., "Glutamate excitotoxicity is involved in cell death caused by tributyltin in cultured rat cortical neurons," Toxicological Sciences, vol. 89, no. 1, pp. 235-242, 2006.

[11] E. V. Jones, D. Cook, and K. K. Murai, "A neuron-astrocyte co-culture system to investigate astrocyte-secreted factors in mouse neuronal development," Methods in Molecular Biology, vol. 814, part 4, pp. 341-352, 2012.

[12] S. Kaech and G. Banker, "Culturing hippocampal neurons," Nature Protocols, vol. 1, no. 5, pp. 2406-2415, 2006.

[13] T. Kaminuma, Y. Suzuki, K. Shirai et al., "Effectiveness of carbon-ion beams for apoptosis induction in rat primary immature hippocampal neurons," Journal of Radiation Research, vol. 51, no. 6, pp. 627-631, 2010.

[14] S. Majd, A. Zarifkar, K. Rastegar, and M. A. Takhshid, "Culturing adult rat hippocampal neurons with long-interval changing media," Iranian Biomedical Journal, vol. 12, no. 2, pp. 101-107, 2008.

[15] H. Yang, R. Cong, L. Na, G. Ju, and S. W. You, "Long-term primary culture of highly-pure rat embryonic hippocampal neurons of low-density," Neurochemical Research, vol. 35, no. 9, pp. 1333-1342, 2010.

[16] S. Shimizu, A. Abt, and O. Meucci, "Bilaminar co-culture of primary rat cortical neurons and glia," Journal of Visualized Experiments, e3257, no. 57, 2011.

[17] M. A. Deli, C. S. Ábrahám, Y. Kataoka, and M. Niwa, "Permeability studies on in vitro blood-brain barrier models: physiology, pathology, and pharmacology," Cellular and Molecular Neurobiology, vol. 25, no. 1, pp. 59-127, 2005.

[18] P. J. Meberg and M. W. Miller, "Culturing hippocampal and cortical neurons," Methods in Cell Biology, vol. 2003, no. 71, pp. 111-127, 2003.

[19] A. M. Taylor, M. Blurton-Jones, S. W. Rhee, D. H. Cribbs, C. W. Cotman, and N. L. Jeon, "A microfluidic culture platform for CNS axonal injury, regeneration and transport," Nature Methods, vol. 2, no. 8, pp. 599-605, 2005.

[20] J. W. Park, B. Vahidi, A. M. Taylor, S. W. Rhee, and N. L. Jeon, "Microfluidic culture platform for neuroscience research," Nature Protocols, vol. 1, no. 4, pp. 2128-2136, 2006. 

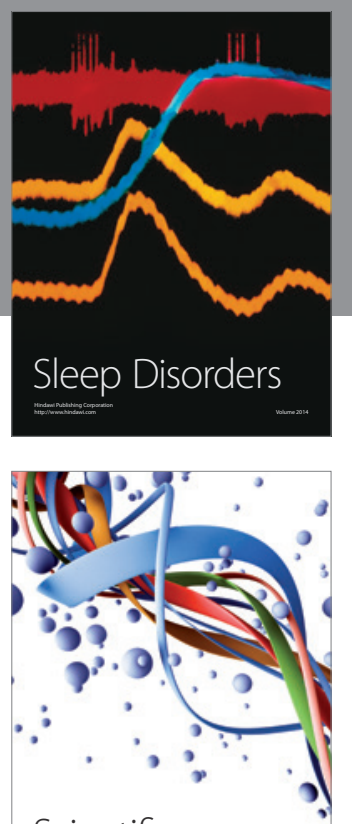

Scientifica
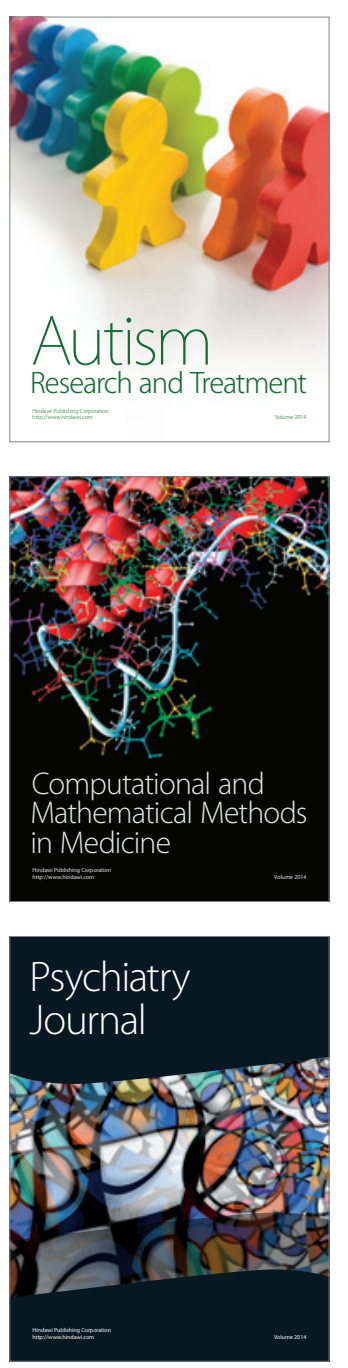
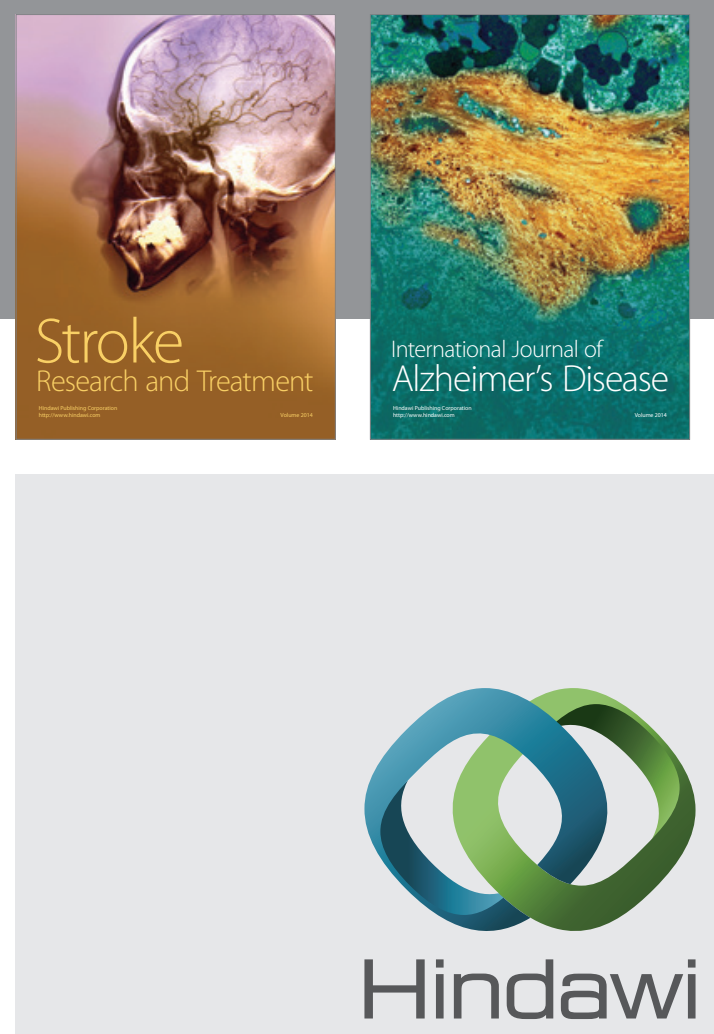

Submit your manuscripts at

http://www.hindawi.com
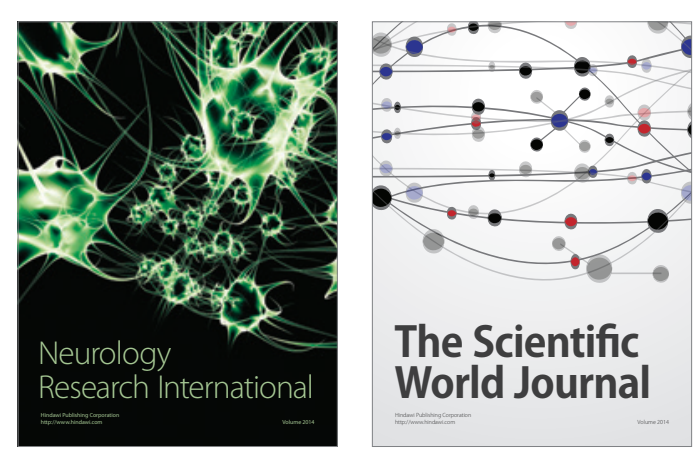

The Scientific World Journal

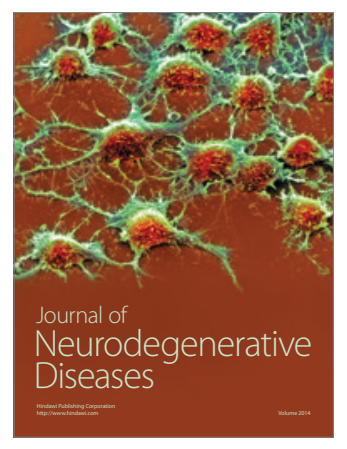

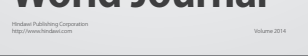

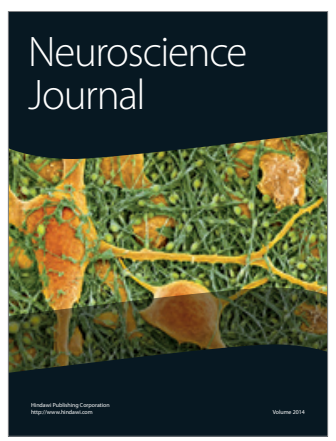

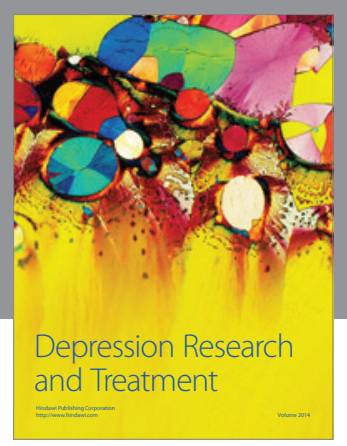
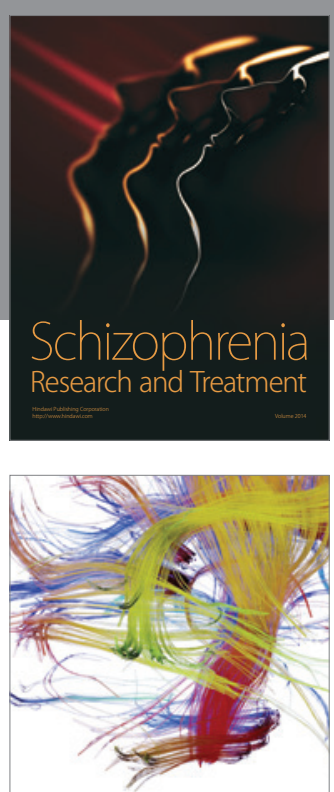

Brain Science

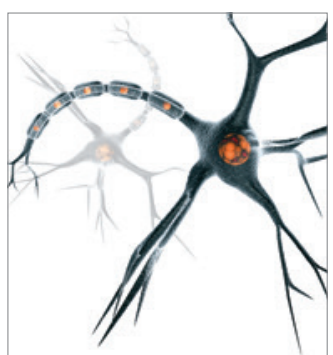

Neural Plasticity
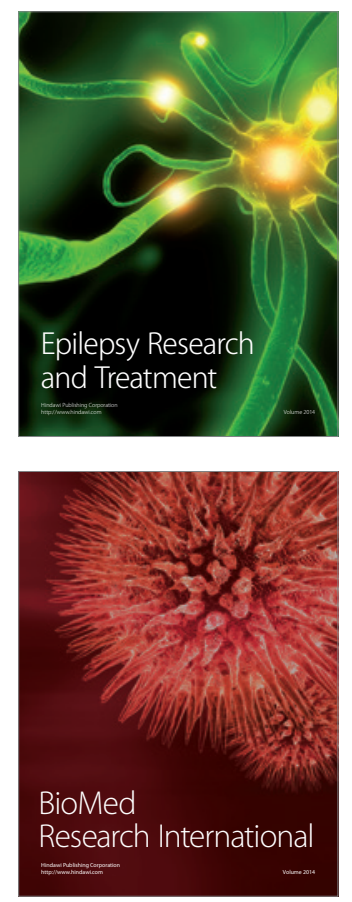

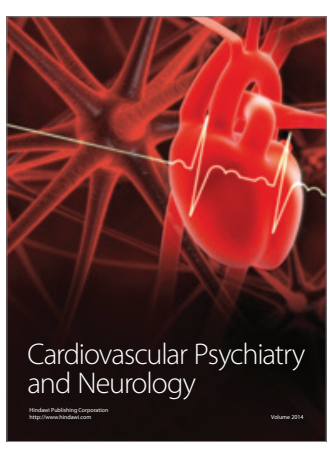

Parkinson's

Disease
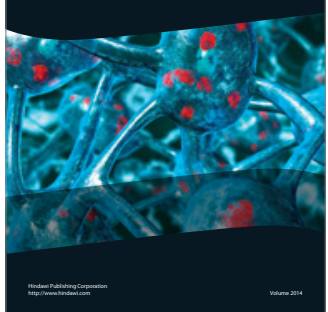